EPJ Web of Conferences 47, 01005 (2013)

DOI: $10.1051 /$ epjconf/20134701005

(C) Owned by the authors, published by EDP Sciences, 2013

\title{
Precision photometry with difference imaging in the WTS
}

\author{
J. Zendejas ${ }^{1,2, a}$, J. Koppenhoefer ${ }^{1}$, R.P. Saglia², J.L. Birkby ${ }^{3}$, S.T. Hodgkin ${ }^{4}$, \\ G. Kovács ${ }^{4}$, D.J. Pinfield ${ }^{5}$, B. Sipőcz ${ }^{5}$ and the RoPACS collaboration \\ ${ }^{1}$ University Observatory Munich, Scheinerstrasse 1, 81679 München, Germany \\ ${ }^{2}$ Max-Planck-Institute for Extraterrestrial Physics, 85748 Giessenbachstrasse, Germany \\ ${ }^{3}$ Leiden Observatory, Leiden University, Niels Bohrweg 2, 2333 CA Leiden, The Netherlands \\ 4 Institute of Astronomy, University of Cambridge, Madingley Road, Cambridge CB3 OHA, UK \\ ${ }^{5}$ Centre for Astrophysics Research, University of Hertfordshire, College Lane, Hatfield, \\ Hertfordshire AL10 9AB, UK
}

\begin{abstract}
The Wide Field Camera Transit Survey is a pioneer program aimed to search for extra-solar planets in the near-infrared. The standard data reduction pipeline of the program uses aperture photometry to construct the light curves. We alternatively apply the difference imaging method for the most complete field in the survey and carry out a quantitative comparison between the photometric precision of both methods. We also report an implementation on the box-fitting detection algorithm, which performs a trapezoid-fit to the folded light curve. Subsequently we apply a set of selection criteria to the light curves to search for transit candidates, incorporating a parameter to characterize the shape of the transit. We carry out a detailed analysis of 11 candidates and provide a classification scheme to separate binary from planet candidates. Furthermore we report the detection of five faint extremely-short period eclipsing binary systems with periods shorter than 0.23 days and one candidate M-dwarf/M-dwarf eclipsing binary.
\end{abstract}

\section{INTRODUCTION}

In recent years several transit missions and surveys have been launched to find and characterize new exo-planets. Among them, the WFCAM ${ }^{1}$ Transit Survey (WTS) is a pioneer project that stands out for its particular aims. The WTS is focused on the discovery of rocky and potentially habitable planets using the transit method for the first time in the near infrared wavelength regime.

Traditionally, aperture photometry (AP) has been the standard technique to produce light curves in transit surveys. In 1996, a new method to study crowded fields by optimal image subtraction was presented by [1]. This method (usually called "difference imaging", DI) was initially developed to study microlensing events in crowded fields. However, since the majority of the transit survey targets are crowded fields, image subtraction photometry has become an important tool to search for planetary transits.

Due to the large number of light curves in transit surveys, an efficient detection algorithm is needed to speed up the identification of planet candidates. For instance, [2] presented a box-fitting algorithm based on least squares fit of step functions (BLS) to analyze stellar photometric time series, which has become a popular tool in the search for exo-planets. Transit surveys additionally require ways to minimize the number of false-positives and facilitate the selection of the best candidates. In this study we

\footnotetext{
${ }^{a}$ e-mail: chicho@usm. uni-muenchen.de

${ }^{1}$ Wide Field Camera, see http://www . jach.hawaii.edu/UKIRT/instruments/wf cam/

This is an Open Access article distributed under the terms of the Creative Commons Attribution License 2.0, which permits unrestricted use, distribution, and reproduction in any medium, provided the original work is properly cited.
} 


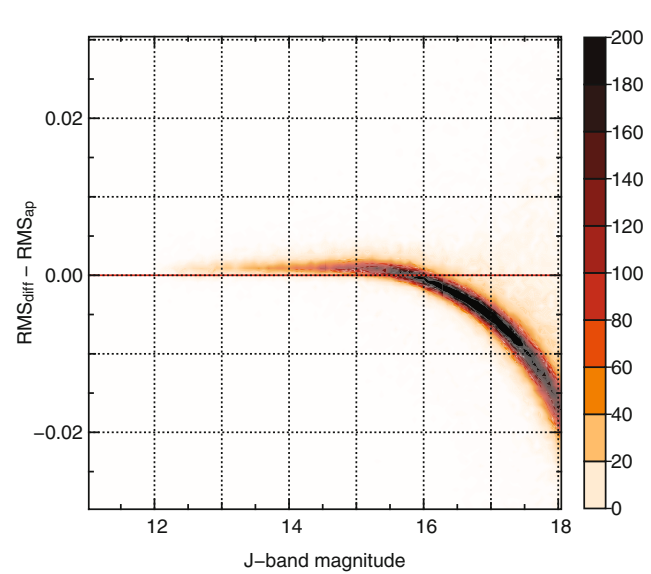

(a)

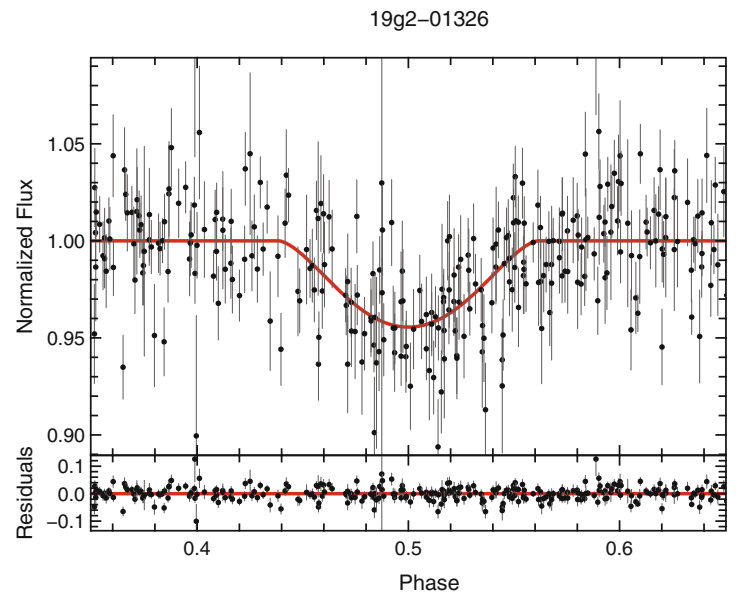

(b)

Figure 1. (a) Difference between the RMS of DI and AP light curves as a function of the J-band magnitude. The plot shows the density of data points distributed in $100 \times 100$ bins. (b) Best fitting model of $19 \mathrm{~g}-2-01326$ using the J-band light curve. The top frame shows the best fit, whereas the bottom frame represents the residuals of the fit.

employ a series of selection criteria to search for transit planets based on previous works and incorporate a new criterion named "V-shape parameter" that is designed to recognize automatically eclipsing binary systems.

\section{DI LIGHT CURVES AND COMPARISON WITH THE AP METHOD}

We use DI photometry to construct an alternative set of the WTS-light curves. In order to show the quality of DI light curves, we carry out a quantitative analysis by comparing the photometric precision (RMS) of the DI and AP light curves. We apply the sysrem algorithm to both sets of light curves to remove systematic effects and correct the error bars of the light curves by using a scale factor, since for many light curves (typically bright sources) the scatter of the data points was much larger than the error bars. This step is important, because we include selection criteria to detect planet candidates that incorporate parameters related directly to the error bars. We present the results of the comparison in Fig. 1(a), which shows that DI light curves produce significantly better results for objects with $\mathrm{J}>16$.

\section{LIGHT CURVE ANALYSIS AND TRANSIT DETECTIONS}

We detect transits in the WTS light curves using an algorithm which is based on the Box-fitting Least Squares (BLS) algorithm that has been proposed by [2]. Our modifications include a trapezoidal refitting of the box-shaped eclipse found by BLS where the re-fitting is done by symmetrically varying the edges of the box while keeping the duration of the eclipse fixed. The shape of the trapezoid provides a new parameter called the $V$-shape, which we use as a criterion to reject grazing eclipsing binary systems that have generally very large $V$-shape values. One of the advantages of our modification is that the $\mathrm{V}$-fitting results in a better estimate of the transit depth.

\section{SELECTION CRITERIA}

Due to the large number of light curves in the WTS, it is necessary to set up selection criteria in order to automatize and speed-up the search of candidates. We use 8 steps as selection criteria to search for 


\section{Hot Planets and Cool Stars}

Table 1. Classification list of candidates. The second column shows the data set in which the candidates have been detected. We show in columns 8-9 the candidate's radii and the corresponding $1 \sigma$ lower limit. Note that the period corresponds to a planet scenario with a single eclipse and some of the candidates classified as eclipsing binaries have actually periods of double the listed value. The J-band magnitude corresponds to the WFCAM measurements in the Vega-system. Last column shows our classification, where P represents a planet candidate and B a binary system.

\begin{tabular}{|c|c|c|c|c|c|c|c|c|c|c|c|}
\hline Object & Pho.Tech & $\alpha$ & $\delta$ & Period(days) & $t_{0}$ & $i\left(^{o}\right)$ & $\mathrm{R}_{\text {planet }}\left(\mathrm{R}_{J u p}\right)$ & $\mathrm{R}_{\text {planet,min }}\left(\mathrm{R}_{\text {Jup }}\right)$ & J-mag & $T_{\text {eff }}$ & Classification \\
\hline $19 \mathrm{~g}-2-01326$ & DI\&AP & 294.2724 & 36.2587 & 0.428 & 2454318.0353534 & 69.0 & 2.29 & 1.68 & 17.04 & 4500 & $\mathrm{P}$ \\
\hline $19 \mathrm{~b}-1-02162$ & DI\&AP & 293.0112 & 36.4848 & 0.599 & 2454317.7883529 & 72.0 & 1.61 & 1.40 & 15.93 & 5500 & B \\
\hline $19 \mathrm{e}-1-01554$ & DI & 292.7598 & 36.1322 & 1.483 & 2454318.8079255 & 83.1 & 2.20 & 2.08 & 17.46 & 4000 & B \\
\hline $19 \mathrm{~d}-4-12670$ & DI\&AP & 294.1152 & 36.9149 & 0.576 & 2454317.9681650 & 76.1 & 2.31 & 2.21 & 17.10 & 4500 & B \\
\hline $19 \mathrm{~h}-3-06876$ & DI & 294.5409 & 36.5086 & 1.190 & 2454318.6883788 & 81.4 & 2.51 & 2.42 & 16.73 & 4000 & B \\
\hline $19 e-3-06726$ & DI\&AP & 293.1551 & 36.6596 & 0.927 & 2454318.0769202 & 80.3 & 2.54 & 2.47 & 17.48 & 4000 & B \\
\hline $19 c-4-06354$ & DI & 293.7592 & 36.8153 & 0.383 & 2454318.0542919 & 65.8 & 3.31 & 2.17 & 17.97 & 3500 & B \\
\hline $19 a-1-01898$ & DI & 292.7391 & 36.3647 & 0.582 & 2454318.0188718 & 69.6 & 3.64 & 2.50 & 17.62 & 3750 & B \\
\hline $19 \mathrm{c}-4-02952$ & $\mathrm{AP}$ & 293.8666 & 36.7571 & 3.430 & 2454319.6314035 & 82.2 & 3.54 & 3.36 & 15.83 & 6250 & B \\
\hline $19 \mathrm{e}-2-02057$ & DI & 293.2809 & 36.2500 & 0.665 & 2454318.3737809 & 69.1 & 4.72 & 2.18 & 17.28 & 5250 & B \\
\hline $19 \mathrm{~g}-1-11212$ & AP & 293.6753 & 36.1420 & 2.773 & 2454318.9644598 & 74.4 & 7.21 & 3.28 & 14.01 & 6250 & B \\
\hline
\end{tabular}

transit planets. Five of them are based on the experience of previous works, such as setting a limit in the brightness of the objects $(\mathrm{J}<18)$, signal-to-noise ratio $(S / N \geq 20)$, number of transit points $(\geq 20)$, transit depth $(\leq 10 \%)$, as well as removing alias periods in the ranges $0.485-0.515,0.985-1.015,1.985$ 2.015 and 2.985-3.015 days. We additionally incorporate 3 criteria defined from the results of our transit detection algorithm. Before describing the first criterion, it is necessary to define the quantity signal to noise removed $(S / N)_{\text {rem }}$. The algorithm selects the best trial period, providing a transit detection for a particular light curve. We then mask the transit and apply again the detection algorithm on the masked light curve. For a planet candidate, we do not expect a second detection, therefore a high $(S / N)_{\text {rem }}$ is an indication of a variable star. We define a selection criterion as the difference between the signal-tonoise and signal-to-noise removed $S / N-S / N_{\text {rem }}$. For a good candidate, we expect a high value of this quantity, otherwise two high signal detection might exist in the light curve. We adopt the limit for this criterion in $S / N-S / N_{\text {rem }} \geq 8$. The two remaining criteria are the $V$-shape parameter $(V \leq 0.75)$ and a cut in the reduced $\chi^{2}$ provided by the trapezoid-fit $(\leq 3.5)$. Detailed information about the selection criteria can be found in [3].

\section{PRESENTATION OF CANDIDATES AND CLASSIFICATION LIST}

Our selection criteria were applied to the DI and AP light curves. 52 objects pass the criteria in the DI data set whereas 38 were detected in the AP light curves. We perform a visual inspection of the 90 detections and keep 11 relevant candidates. So far, two hot-Jupiter planets have been found in the WTS ([4] \& [5]) and one of them passes our selection criteria, however it is not reported in this work. We carry out a detailed analysis of the 11 selected candidates which includes a characterization of the host stars by a Spectral Energy Distribution analysis, a realistic transit fit model [6] and a fit of secondary eclipse for binary scenarios. This analysis provides important physical parameters of the host companions and planet candidates. We use this information to characterize the system and provide a classification list of candidates. The candidates are classified as planet (P) candidate and eclipsing binary system (B) according to the parameters found during the analysis. We present in table 1 the classification list, the measured Jmag and estimated temperature of the host stars, some parameters of the orbit (period and inclination) and the estimated radius of the transiting objects. Figure 1(b) shows the DI light curve together with the best fitting transit model for our planet candidate.

\section{OTHER APPLICATIONS OF WTS DI LIGHT CURVES}

The DI light curves can be used for additional analyses, such as detection and characterization of faint variable stars. Recently, [7] reported a sample of 15 eclipsing binaries from the WTS-19hr field with periods shorter than 0.3 days. We ran our detection algorithm on the DI light curves and we found 


\section{EPJ Web of Conferences}

additionally 5 eclipsing binaries with periods shorter than 0.23 days that were not previously reported. On the other hand, [8] reported the detection of $16 \mathrm{M}$-dwarf eclipsing binary systems (MEBs) with $\mathrm{J}<16$ mag found in the WTS AP light curves. We investigate the potential of extending the search for MEBs to fainter systems with magnitudes $\mathrm{J} \leq 18$ mag making use of the improvement in the photometric precision of DI light curves. We present the object 19c-4-06354 from our classification list as a high potential candidate of being an MEB.

\section{CONCLUSIONS}

We carried out a quantitative comparison between the photometric precision of the AP and DI light curves from the $19 \mathrm{hr}$ field. The results show that the DI light curves present a significant improvement of about 2-20 mmag for sources with J-band magnitudes between 16 and 18 .

A modified version of the box-fitting algorithm was employed to search for transiting planets. Our algorithm includes a trapezoid re-fit of the folded light curve and also calculates a new parameter based on the geometry of the new trapezoid fit, the $V$-parameter. In order to select our candidates, we used a series of selection criteria based on previous works and the results of our detection algorithm. 90 candidates were found in the DI and AP light curves from a original sample of $\sim 475000$ sources.

We conducted a detailed analysis on 11 relevant candidates and presented a classification list of candidates. No planet candidates were found orbiting an $\mathrm{M}$-dwarf with magnitudes $\mathrm{J} \leq 17$, we therefore confirmed the null detection and the sensitivity analysis of WTS presented in [9].

We reported other applications of the WTS DI light curves, such as the detection of five new ultrashort period eclipsing binaries and a detached M-dwarf eclipsing binary candidate. In conclusion, the DI-light curves are useful for many purposes such as detection of transit planet candidates and rare eclipsing binary systems, especially when pushing the limits to fainter magnitudes.

\section{References}

[1] A. B. Tomaney, A. P. S. Crotts, AJ 112, 2872 T (1996)

[2] G. Kovács, S. Zucker, T. Mazeh, A\&A 391, 369K (2002) B. A. McLeod, K. Z. Stanek, J. A. Barranco, M. H. Pinsonneault, S. Meibom, J. S. Kalirai, ApJ 695, 336H (2009)

[3] J. Zendejas, J. Koppenhoefer, R. P. Saglia, et al. (in prep.)

[4] M. Cappetta, R. P Saglia, J. L Birkby, MNRAS 427, 1877C (2012)

[5] Birkby et al. (in prep.)

[6] K. Mandel, E. Agol, ApJ 580L, 171M (2002)

[7] S. V. Nefs, J. L. Birkby, I. A. G. Snellen, S. T. Hodgkin, D. J. Pinfield, B. Sipőcz, G. Kovacs, D. Mislis, R. P. Saglia, J. Koppenhoefer, et al., MNRAS 425, 950N (2012)

[8] J. Birkby, B. Nefs, S. Hodgkin, G. Kovács, B. Sipőcz, D. Pinfield, I. Snellen, D. Mislis, F. Murgas, N. Lodieu, et al., MNRAS 426, 1507B (2012)

[9] G. Kovacs, S. Hodgkin, B. Sipőcz, D. J. Pinfield, et al. (submitted to MNRAS) 